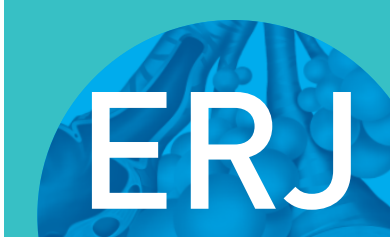

open research

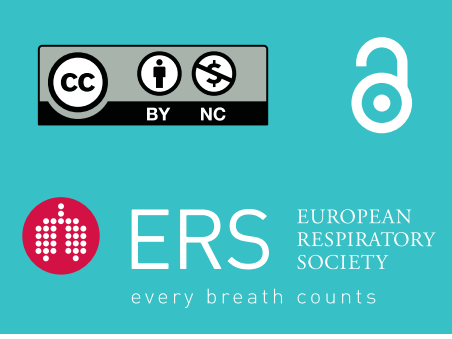

\section{A review of physiotherapy practice for people with bronchiectasis}

\section{To the Editor:}

People with bronchiectasis experience chronic productive cough and acute exacerbations, which are linked to poorer quality of life and a higher rate of disease progression. The clinical guidelines for bronchiectasis management recommend physiotherapy [1,2]. These guidelines advise the prescription of airway clearance techniques (ACTs) to improve sputum clearance and reduce symptoms $[1,2]$. Mucoactive agents may be cautiously considered when required [1,2]. Individuals with reduced exercise tolerance are recommended to engage in pulmonary rehabilitation or undertake exercise or physical activity $[1,2]$. Surveys of physiotherapy management for people with bronchiectasis have described commonly applied ACTs $[3,4]$ but have not alluded to technique combinations, a strategy considered a key approach towards personalising therapy [3-5]. Other audits have highlighted that ACTs were applied in only $49 \%$ and $52 \%$ of people with bronchiectasis in Europe and Australia respectively, reflecting a potential underuse of this therapeutic approach $[6,7]$. Low referral rates to pulmonary rehabilitation have been noted $[3,7]$, and the prescription of exercise and physical activity beyond pulmonary rehabilitation is unknown [3].

It is acknowledged that comorbidities are common in people with bronchiectasis $[1,2]$. Gastro-oesophageal reflux disease (GORD) may increase exacerbation risk [8]. Stress urinary incontinence has been linked to reduced quality of life [9]. Chronic rhinosinusitis is associated with greater disease severity and poorer HRQOL [10]. Those with bronchiectasis may be at an increased risk of cardiovascular disease, independent of established cardiovascular risk factors or comorbidities [11]. Finally, musculoskeletal disorders including osteoarthritis or osteoporosis have been noted in bronchiectasis, and may account for joint stiffness and pain [12].

These comorbidities may influence the ACT or exercise prescription, or require specific physiotherapy to alleviate symptoms. However, there is no understanding of how frequently these comorbidities are assessed by physiotherapists and what clinical modifications are incorporated to manage these comorbidities. This audit sought to explore the current physiotherapy practice for ACTs, pulmonary rehabilitation and physiotherapy approaches for comorbidity management in people with bronchiectasis within a private healthcare facility.

For a 12-month period (June 2018-2019), an audit of 56 participants diagnosed with bronchiectasis who received outpatient physiotherapy at Cabrini Health, Melbourne, Australia during this time frame was conducted. There were no exclusions for data incompleteness. Assessment of current symptoms, clinical status and presence of comorbidities were extracted from a single occasion of service (the initial consultation). The ACT routine, recommendations for exercise prescription (including pulmonary rehabilitation referral) and management strategies for comorbidities were extracted from medical records by a single investigator using an a priori template. Approval was obtained from the Cabrini Health Human Research Ethics Committee.

The mean \pm SD age of participants was $76 \pm 11$ years with a median of 2 (interquartile range 1-4) exacerbations in the year preceding the initial consultation. All were referred by respiratory physicians. During this timeframe, $96 \%(n=54)$ were referred for ACTs, while one participant was referred for haemoptysis and one for exercise prescription. With regards to clinical review, chest status assessment incorporated history and status of cough and sputum production, breathlessness, and existing ACT or

@ERSpublications

A combination of airway clearance techniques are applied for people with bronchiectasis, together with recommendations for exercise and suggestions for management of common comorbidities https://bit.ly/2U3c99H

Cite this article as: Lee AL, Baenziger S, Louey A, et al. A review of physiotherapy practice for people with bronchiectasis. ERJ Open Res 2021; 7: 00569-2020 [https://doi.org/10.1183/ 23120541.00569-2020].

Copyright $\odot$ ERS 2021. This article is open access and distributed under the terms of the Creative Commons Attribution NonCommercial Licence 4.0. 


\begin{tabular}{|c|c|}
\hline & Participants \\
\hline \multicolumn{2}{|l|}{ ACT } \\
\hline PEP therapy & $1(2)$ \\
\hline ACBT & $36(64)$ \\
\hline Positioning & $10(18)$ \\
\hline Oscillating PEP therapy & $29(52)$ \\
\hline Saline ${ }^{\#}$ inhalation & $30(54)$ \\
\hline Exercise for ACT & 22 (39) \\
\hline \multicolumn{2}{|l|}{ Common combinations of ACTs } \\
\hline ACBT, inhaled saline and exercise & 5 (9) \\
\hline ACBT, inhaled saline and oscillating PEP therapy & $6(11)$ \\
\hline Oscillating PEP therapy, inhaled saline and exercise & 5 (9) \\
\hline ACBT and exercise & 5 (9) \\
\hline ACBT and oscillating PEP therapy & 5 (9) \\
\hline \multicolumn{2}{|l|}{ Extrapulmonary management strategies } \\
\hline Thoracic mobility exercises for pain & $2(4)$ \\
\hline Suggestions for optimising GORD management" & 7 (13) \\
\hline Sinus rinsing and nasal hygiene & $8(14)$ \\
\hline Postural exercises & $18(32)$ \\
\hline Techniques for breathing dysfunction and phonation & $5(9)$ \\
\hline
\end{tabular}

exercise routine, if applicable. A valid measure of exercise tolerance, lower limb strength or functional mobility was completed in $33 \%$ of participants; tools included the 6 -min walk test $(2 \%)$, 10 -m walk test (3\%) and sit-to-stand test (18\%). Shortness of breath was assessed in $86 \%$ of participants, with a self-reported description applied in 94\%. Upper airway symptoms (rhinosinusitis) were assessed in 39\% of participants, incontinence was assessed in 9\%, GORD was assessed in $88 \%$ and musculoskeletal pain was assessed in $32 \%$.

All participants were instructed in ACTs by a clinically experienced physiotherapist. 12 participants (21\%) used a single technique, $36 \%$ used two techniques and $43 \%$ used three or more techniques, with proportions of use and common combinations outlined in table 1. Treatment approaches for extrapulmonary symptoms were documented for $23 \%$ of participants. Thoracic and shoulder mobility exercises were prescribed for pain or postural dysfunction. A mix of suggestions to address GORD, including nonprescription medications, physical modalities, and advice for timing of ACTs and oral intake, were prescribed. Treatment for rhinosinusitis consisted of sinus rinsing and upper airway humidification or nebulisation. Techniques to counter breathing dysfunction were prescribed in a small number of participants.

19 participants (29\%) were referred to pulmonary rehabilitation while $57 \%$ were recommended to undertake other exercise, including walking (63\%), exercise bike (9\%), yoga (4\%), dancing, golf or Pilates (each 2\%). A small proportion of participants (11\%) were recommended a combination of physical activities.

We found a mix of ACTs were applied and prescribing a combination of ACTs was the most common approach. This strategy is considered an acceptable method, personalising ACT while considering physiological parameters, symptoms and patient preference [5]. The frequent selection of the active cycle of breathing technique (ACBT), oscillating positive expiratory pressure (PEP) therapy and exercise in isolation or combination in this study aligns with practice recommendations [1] and surveys [3, 13]. While there is evidence supporting the role of ACBT and oscillating PEP therapy in bronchiectasis [1, 2], the effect of exercise as an ACT is unclear. Despite this, the application of exercise as an ACT within this audit and a survey conducted in 2016 [13] highlight the need to establish the benefits of this technique in bronchiectasis. The caution in guidelines related to inhaled saline therapy is predominantly restricted to hypertonic saline $[1,2]$. It was not consistently clear from this audit whether hypertonic or isotonic saline was prescribed. However, its regular use is supported by the suggestion to consider a trial of mucoactive therapy for individuals experiencing difficulty in secretion clearance $[1,2]$. 
Formal appraisal of exercise tolerance or exertional breathlessness was limited. However, only a single session of physiotherapy was audited and with time constraints, prioritisation of assessment and treatment are necessary. The encouragement of individuals to engage in different forms of exercise aligns with clinical guidelines $[1,2]$. However, with a mix of exercise modes, the intensity of this exercise is likely to be variable. Incorporating a regular, validated measure of exercise capacity within a clinical review provides the opportunity for specific exercise prescription. Only a small proportion were referred to pulmonary rehabilitation. Pulmonary rehabilitation guidelines indicate the inclusion of those with bronchiectasis with symptoms of breathlessness [14]. The predominant use of subjective descriptions of breathlessness over objective measures may limit the understanding of symptom behaviour and the extent to which exercise intolerance is present. This, together with the high portion of individuals prescribed walking as a main form of exercise, may account for the low referral rate.

Enquiry regarding comorbidities was largely variable but is likely to be influenced by patients' clinical presentation and assessment priorities. The range of treatment applied aligns with physiotherapy approaches for these comorbidities in individuals with cystic fibrosis [15]. This audit provides some guidance regarding what approaches require further investigation to determine their therapeutic worth and provide advice for physiotherapy treatments in the future.

In conclusion, combinations of ACTs were applied for people with bronchiectasis, together with recommendations for exercise. Future research could examine the effect of exercise as an ACT in people with bronchiectasis, including the impact on patient-reported outcomes, and explore the clinical benefits of physiotherapy-directed advice or treatment for managing comorbidities.

Annemarie L. Lee $\oplus^{1,2,3}$, Susy Baenziger ${ }^{4}$, Amanda Louey ${ }^{4}$, Sophie Jennings $\oplus^{1,4}$, Peter Solin ${ }^{4}$ and Ryan Hoy ${ }^{4,5}$

${ }^{1}$ Centre for Allied Health Research and Education, Cabrini Health, Malvern, Australia. ${ }^{2}$ Dept of Physiotherapy, Monash University, Melbourne, Australia. ${ }^{3}$ Institute for Breathing and Sleep, Austin Health, Melbourne, Australia. ${ }^{4}$ Cabrini Health, Malvern, Australia. ${ }^{5}$ Allergy, Asthma and Clinical Immunology, Alfred Health, Melbourne, Australia.

Correspondence: Annemarie L. Lee, Centre for Allied Health Research and Education, Cabrini Institute, 154 Wattletree Road, Malvern, 3144, Victoria, Australia. E-mail: AnnemarieLee@cabrini.com.au

Received: 12 Aug 2020 | Accepted: 1 Oct 2020

Conflict of interest: None declared.

\section{References}

1 Hill A, Sullivan A, Chalmers J, et al. British Thoracic Society guideline for bronchiectasis in adults. Thorax 2019; 74: 1-69.

2 Polverino E, Goeminne P, O’Donnell M, et al. European Respiratory Society guidelines for the management of adult bronchiectasis. Eur Respir J 2017; 50: 1700629.

3 Lee A, Button B, Denehy L. Current Australian and New Zealand physiotherapy practice in the management of patients with bronchiectasis and COPD. NZ J Physiother 2008; 36: 49-58.

4 O'Neill B, Bradley J, McArdle N, et al. The current physiotherapy management of patients with bronchiectasis: a UK survey. Int J Clin Pract 2002; 56: 34-35.

5 McIlwaine M, Bradley J, Elborn J, et al. Personalising airway clearance in chronic lung disease. Eur Respir Rev 2017; 26: 160086.

6 Cortina B, Aliberti S, Blasi F, et al. Chest physiotherapy in European patients with bronchiectasis: data from the EMBARC registry. Eur Respir J 2017; 50: A4071.

7 Visser SK, Bye PTP, Fox GJ, et al. Management of Australian Adults with Bronchiectasis in Tertiary Care: Evidence-based or access driven? Lung 2019; 197: 803-810.

8 Mandal P, Morice A, Chalmers J, et al. Symptoms of airway reflux predict exacerbations and quality of life in bronchiectasis. Respir Med 2013; 107: 1008-1013.

9 Duignan N, McDonnell M, Mokoka M, et al. High prevalence of stress urinary incontinence in adult patients with bronchiectasis. Ir Med J 2016; 109: 440.

10 Handley E, Nicolson C, Hew M, et al. Prevalence and clinical implications of chronic rhinosinusitis in people with bronchiectasis: a systematic review. J Allergy Clin Immunol Pract 2019; 7: 2004-2012.

11 Navaratnam V, Millett ERC, Hurst JR, et al. Bronchiectasis and the risk of cardiovascular disease: a population based study. Thorax 2017; 72: 161-166.

12 Gale N, Bolton C, Duckers JM, et al. Systemic comorbidities in bronchiectasis. Chron Respir Dis 2012; 9: 231-238

13 Phillips J, Lee AL, Pope R, et al. Current Australian and New Zealand physiotherapy practices during an acute exacerbation of bronchiectasis. Respirology 2019; 24: TP122.

14 Alison JA, McKeough ZJ, Johnston K, et al. Australian and New Zealand Pulmonary Rehabilitation Guidelines. Respirology 2017; 22: 800-819.

15 Button BM, Wilson C, Dentice R, et al. Physiotherapy for cystic fibrosis in Australia and New Zealand: a clinical practice guideline. Respirology 2016; 21: 656-667. 